\title{
Discontinuous Petrov-Galerkin Methods for Topology Optimization
}

\author{
Evgrafov, Anton
}

Published in:

EngOpt 2018 Proceedings of the 6th International Conference on Engineering Optimization

Link to article, DOI:

10.1007/978-3-319-97773-7_24

Publication date:

2018

Document Version

Peer reviewed version

Link back to DTU Orbit

Citation (APA):

Evgrafov, A. (2018). Discontinuous Petrov-Galerkin Methods for Topology Optimization. In EngOpt 2018

Proceedings of the 6th International Conference on Engineering Optimization (pp. 260-271). Springer Publishing Company. https://doi.org/10.1007/978-3-319-97773-7_24

\section{General rights}

Copyright and moral rights for the publications made accessible in the public portal are retained by the authors and/or other copyright owners and it is a condition of accessing publications that users recognise and abide by the legal requirements associated with these rights.

- Users may download and print one copy of any publication from the public portal for the purpose of private study or research.

- You may not further distribute the material or use it for any profit-making activity or commercial gain

- You may freely distribute the URL identifying the publication in the public portal

If you believe that this document breaches copyright please contact us providing details, and we will remove access to the work immediately and investigate your claim. 


\title{
Discontinuous Petrov-Galerkin methods for topology optimization
}

\author{
Anton Evgrafov ${ }^{1,2 \star \star}$ \\ 1 Department of Mathematical Sciences, \\ Norwegian University of Science and Technology, N-7491 Trondheim, Norway \\ 2 Department of Mechanical Engineering, \\ Technical University of Denmark, DK-2800 Kgs. Lyngby, Denmark, \\ aaev@mek.dtu.dk
}

\begin{abstract}
Discontinuous Petrov-Galerkin (DPG) methods constitute a modern class of finite element methods, which present several advantages when compared with traditional Bubnov-Galerkin methods, especially when the latter is applied to indefinite or non-symmetric problems. Our objective is to utilize the advantages of DPG methods in the context of topology optimization.

The direct application of DPG discretizations to BVPs arising in topology optimization is hindered by the very unusual scaling of the residual, caused by the gigantic jumps in the coefficients of the governing differential equations. In the prototypical case of linearized elasticity with SIMP model the coefficient ratio between the "stiff" and "soft" phases is held at a billion, which is further squared by Petrov-Galerkin methods based on minimizing the squared residual.

We introduce a DPG method with appropriately scaled residual norm, which allows us to deal with big contrast ratios in the coefficients. The method is tested on benchmark topology optimization problems.
\end{abstract}

Keywords: Discontinuous Petrov-Galerkin methods, DPG, topology optimization

\section{Introduction}

Most commonly used topology optimization algorithms are based on repeatedly solving discretized boundary value problems (BVPs) for the governing partial differential equations (PDEs) and their adjoints corresponding to a given design approximation, which is then updated in accordance with the outcome of these computations, see for example $[1,4]$. Computational burden associated with repeatedly solving these algebraic equations often completely dominates over the rest of the computations, and it is only natural to seek ways for reducing it. For large scale problems the BVPs are solved using iterative methods, typically Krylov subspace algorithms such as for example the preconditioned conjugate

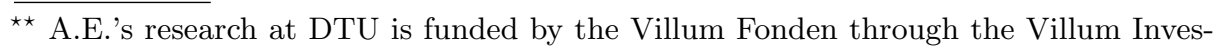
tigator Project InnoTop. 
gradient (PCG) method. One line of reasoning is therefore to stop these iterative methods prior to their successful termination according to some conservative stopping criterion. Such a strategy, based on various heuristic stopping criteria, has been pursued in for example [2]. In [12] it has been proposed to stop the preconditioned Krylov iteration when the residual of the governing or adjoint PDEs, as estimated by a posteriori analysis, is dominated by the discretization error. Such a situation has likely occurred when the residual estimate is no longer being reduced as Lanczos or Arnoldi iteration progresses. It turns out that for some representative benchmarks topology optimization problems in linear elasticity this stopping criterion is satisfied already after only a few Krylov subspace iterations, yet the overall optimization progress is practically not affected by such an early termination of the method. The downside of this strategy is that in addition to a matrix-vector multiplication, one has to carry out an a posteriori error estimation analysis in each iteration of a Krylov subspace algorithm. Depending on how this is done, the associated computational cost could be comparable with or exceeding that needed to solve the governing equations in the first place.

In order to deal with this conundrum, we investigate the possibility of utilizing discontinuous Petrov-Galerkin (DPG) methods [7] for discretizing the governing and adjoint BVPs arising in the topology optimization problems. Postponing a detailed discussion of this class of methods, we note that evaluating the residual estimate within DPG framework amounts to solving small elementwisedecoupled variational problems and integration, all of which can be done in an embarrassingly parallel fashion.

Our main finding is that whereas the naive application of DPG methods within the topology optimization framework for linearized elasticity leads to erroneous results, a simple scaling of the test space norm recovers the convergence properties of this class of finite elements methods for the unusually scaled PDEs in topology optimization.

\section{Brief statement of the optimization problem}

We consider the standard problem of compliance minimization based on a SIMP material model, where the material distribution enters the governing equations of linearized elasticity through the application of regularizing inverse Helmholtz operator (a.k.a. "density filtering"). Namely, let $\Omega \subset \mathbb{R}^{n}, n \in\{2,3\}$ be an open bounded domain with Lipschitz boundary $\Gamma=\partial \Omega$. We assume that $\Gamma$ is decomposed into disjoint open parts $\Gamma_{D}, \Gamma_{N} \subset \Gamma, \overline{\Gamma_{D} \cup \Gamma_{N}}=\Gamma$ where the Dirichlet (displacement) and Neumann (traction) boundary conditions will be imposed.

Given a positive number $r>0$ we denote by $\mathcal{F}_{r}: L^{2}(\Omega) \rightarrow L^{2}(\Omega)$ the compact linear "filtering" operator $\mathcal{F}_{r}=\left(I-r^{2} \Delta\right)^{-1}$, where $I$ is the identity operator and homogeneous Neumann boundary conditions $\partial_{n} \rho=0$ are assumed 
on $\Gamma$.The behaviour of the elastic body is governed by the following BVP:

$$
\begin{aligned}
& -\operatorname{div} \boldsymbol{\sigma}=\mathbf{f}, \quad \text { in } \Omega, \quad \hat{\mathbf{n}} \cdot \boldsymbol{\sigma}=\hat{\mathbf{t}}, \quad \text { on } \Gamma_{N}, \\
& \mathbf{S}_{\varrho} \boldsymbol{\sigma}-\boldsymbol{\varepsilon}(\mathbf{u})=0, \quad \text { in } \Omega, \quad \mathbf{u}=\hat{\mathbf{u}}_{0}, \quad \text { on } \Gamma_{D},
\end{aligned}
$$

where $(\boldsymbol{\sigma}, \mathbf{u}) \in H\left(\Omega, \operatorname{div} ; \mathbb{S}^{n}\right) \times H^{1}\left(\Omega ; \mathbb{R}^{n}\right)$ are the stresses and the displacements of the system, $\boldsymbol{\varepsilon}(\mathbf{u})=\left[\nabla \mathbf{u}+(\nabla \mathbf{u})^{\mathrm{T}}\right] / 2 \in L^{2}\left(\Omega ; \mathbb{S}^{n}\right)$ are the linearized strains, $\mathbf{f} \in L^{2}\left(\Omega ; \mathbb{R}^{n}\right)$ and $\hat{\mathbf{t}} \in L^{2}\left(\Gamma_{N} ; \mathbb{R}^{n}\right)$ are the volumetric and traction forces, $\hat{\mathbf{u}}_{0} \in$ $H^{1 / 2}\left(\Gamma_{D}\right)$ are the prescribed displacements, $\hat{\mathbf{n}}$ is the outwards facing unit normal on $\Gamma$, and $\mathbb{S}^{n}$ is the set of symmetric $n \times n$ second order tensors equipped with Frobenius inner product. $\mathbf{S}_{\varrho} \in L^{\infty}\left(\Omega ; \mathcal{L}\left(\mathbb{S}^{n} ; \mathbb{S}^{n}\right)\right)$ is a spatially varying fourth order compliance tensor, which is assumed to be uniformly bounded and positive definite. Its dependence on the material distribution satisfies the usual SIMP model $\mathbf{S}_{\varrho}=\left(\varrho^{p} \mathbf{C}_{1}\right)^{-1}$, where $p \geq 1$ is the penalty factor (typically $p=3$ in practical computations) and $\mathbf{C}_{1}$ is the stiffness tensor corresponding to the stiff material defined as $\mathbf{C}_{1} \varepsilon=\frac{E}{1+\nu} \varepsilon+\frac{E \nu}{1-\nu^{2}} \operatorname{trace}(\varepsilon) I$, where $I$ is the identity tensor, $E$ is the Young's modulus, and $\nu$ is the Poisson's ratio.

Compliance topology optimization problem in this notation can be stated as follows:

$$
\begin{aligned}
\operatorname{minimize}_{\rho \in L^{2}(\Omega)} J(\rho)= & \int_{\Omega} \mathbf{f}(x) \cdot \mathbf{u}_{\mathcal{F}_{r} \rho}(x) \mathrm{d} x+\int_{\Gamma_{N}} \hat{\mathbf{t}}(x) \cdot \mathbf{u}_{\mathcal{F}_{r} \rho}(x) \mathrm{d} x \\
& +\int_{\Gamma_{D}}\left(\boldsymbol{\sigma}_{\mathcal{F}_{r} \rho}(x) \hat{\mathbf{n}}(x)\right) \cdot \hat{\mathbf{u}}_{0}(x) \mathrm{d} x, \\
\text { s.t. } \quad & V(\rho)=\int_{\Omega} \rho(x) \mathrm{d} x \leq V_{0}, \\
& 0<\rho_{\min } \leq \rho(x) \leq 1, \quad \text { a.e. in } \Omega,
\end{aligned}
$$

where $\rho_{\min } \in(0,1)$, and $V_{0}>\rho_{\min }|\Omega|$ are given. The pair of functions $\left(\boldsymbol{\sigma}_{\mathcal{F}_{r} \rho}, \mathbf{u}_{\mathcal{F}_{r} \rho}\right)$ is the unique solution to (1) corresponding to the material distribution $\varrho=\mathcal{F}_{r} \rho$. The gradient of $J$ with respect to $\rho$ is classically computed as

$$
\nabla_{\rho} J=-\mathcal{F}_{r}\left[p \rho^{-p-1} \mathbf{C}_{1}^{-1} \boldsymbol{\sigma}_{\mathcal{F}_{r} \rho}: \boldsymbol{\sigma}_{\mathcal{F}_{r} \rho}\right],
$$

where we do not need to involve adjoint equations because the problem is selfadjoint. In this work this gradient is then utilized in the optimality criterion (OC) update scheme, but of course could also be used in the context of any other non-linear constrained optimization algorithm. Within the OC scheme, given a current material distribution $\rho_{k}$ we first compute $\left(\boldsymbol{\sigma}_{\mathcal{F}_{r} \rho_{k}}, \mathbf{u}_{\mathcal{F}_{r} \rho_{k}}\right)$ by (approximately) solving the elasticity equations, and then the corresponding gradient $\nabla_{\rho} J_{k}$ from (3). The new material distribution is defined by a simple pointwise update scheme

$$
\rho_{k+1}=\pi_{B_{k}}\left[\rho_{k}\left(-\nabla_{\rho} J_{k} / \lambda_{k+1}\right)^{\xi}\right],
$$

where $\pi_{B_{k}}[\cdot]$ is a projection operator onto a closed, convex, and non-empty set $B_{k}=\left\{\rho \in L^{2}(\Omega) \mid \max \left[\rho_{\min },(1-\gamma) \rho_{k}\right] \leq \rho \leq \min \left[1,(1+\gamma) \rho_{k}\right]\right\}$, and $\gamma>0$ and $\xi \in(0,1)$ are trust-region like and damping parameters, respectively. Finally, $\lambda_{k+1}$ is computed by finding the root of the equation $\int_{\Omega} \rho_{k+1}(x) \mathrm{d} x=V_{0}$ using, for example, the bisection algorithm. For more details see [4]. 


\section{Discontinuous Petrov-Galerkin discretization of (1)}

DPG discretization of the governing equations (1) can be derived as follows, see e.g. [6], even though there are many other possibilities [10]. Let $\Omega_{h}$ be a decomposition of $\Omega$ into shape-regular polygons (elements), $h=\max \{\operatorname{diam}(K) \mid$ $K \in \Omega_{h}$ \}. Further, let $\Gamma_{h}=\cup_{K \in \Omega_{h}} \partial K$ be the skeleton of the mesh. Consider a trial function $U=(\boldsymbol{\sigma}, \mathbf{u}, \hat{\boldsymbol{\sigma}}, \hat{\mathbf{u}}) \in \mathcal{U}=L^{2}\left(\Omega ; \mathbb{R}^{n}\right) \times L^{2}\left(\Omega ; \mathbb{S}^{n}\right) \times H^{-1 / 2}\left(\Gamma_{h} ; \mathbb{R}^{n}\right) \times$ $H^{1 / 2}\left(\Gamma_{h} ; \mathbb{R}^{n}\right)$, and a test function $V=(\boldsymbol{\tau}, \mathbf{v}) \in \mathcal{V}=H\left(\Omega_{h}, \operatorname{div} ; \mathbb{S}^{n}\right) \times H^{1}\left(\Omega_{h} ; \mathbb{R}^{n}\right)$. Boundary conditions can be enforced in DPG in several different ways, and we enforce them as "essential" boundary conditions by considering an affine subspace $\mathcal{U}_{\hat{\mathbf{t}}, \hat{\mathbf{u}}_{0}}$ of $\mathcal{U}$ defined as $L^{2}\left(\Omega ; \mathbb{R}^{n}\right) \times L^{2}\left(\Omega ; \mathbb{S}^{n}\right) \times H_{\hat{\mathbf{t}}}^{-1 / 2}\left(\Gamma_{h} ; \mathbb{R}^{n}\right) \times H_{\hat{\mathbf{u}}}^{1 / 2}\left(\Gamma_{h} ; \mathbb{R}^{n}\right)$ where $H_{\hat{\mathbf{t}}}^{-1 / 2}\left(\Gamma_{h} ; \mathbb{R}^{n}\right)=\left\{\hat{\boldsymbol{\sigma}} \in H^{-1 / 2}\left(\Gamma_{h} ; \mathbb{R}^{n}\right) \mid \hat{\boldsymbol{\sigma}}=\hat{\mathbf{t}}\right.$ on $\left.\Gamma_{N}\right\}$, and $H_{\hat{\mathbf{u}}}^{1 / 2}\left(\Gamma_{h} ; \mathbb{R}^{n}\right)=$ $\left\{\hat{\mathbf{u}} \in H^{1 / 2}\left(\Gamma_{h} ; \mathbb{R}^{n}\right) \mid \hat{\mathbf{u}}=\hat{\mathbf{u}}_{0}\right.$ on $\left.\Gamma_{D}\right\}$.

Let us define the bilinear form $b: \mathcal{U} \times \mathcal{V} \rightarrow \mathbb{R}$ and the linear functional $\ell: \mathcal{V} \rightarrow \mathbb{R}$ corresponding to the ultra-weak formulation of (1), which is obtained in the standard fashion by multiplying the equations with test functions and integrating by parts over each element:

$$
\begin{aligned}
b(U, V)= & \sum_{K \in \Omega_{h}}\left[(\boldsymbol{\sigma}, \boldsymbol{\varepsilon}(\mathbf{v}))_{K}+\left(\mathbf{S}_{\varrho} \boldsymbol{\sigma}, \boldsymbol{\tau}\right)_{K}+(\mathbf{u}, \operatorname{div} \boldsymbol{\tau})_{K}\right. \\
& \left.-\left(\hat{\mathbf{n}}_{\Gamma_{h}} \cdot \hat{\mathbf{n}}_{\partial K}\right)\langle\hat{\boldsymbol{\sigma}}, \mathbf{v}\rangle_{\partial K}-\left\langle\boldsymbol{\tau} \hat{\mathbf{n}}_{\partial K}, \hat{\mathbf{u}}\right\rangle_{\partial K}\right], \\
\ell(V)= & (\mathbf{f}, \mathbf{v})_{\Omega_{h}},
\end{aligned}
$$

where we denote by $(\cdot, \cdot)_{S}$ the $L^{2}(S)$-inner product, and by $\langle\cdot, \cdot\rangle_{S}$ the dual pairing between $H^{-1 / 2}(S)$ and $H^{1 / 2}(S)$. Additionally, we utilize the notation $\hat{\mathbf{n}}_{\partial K}$ for the outwards facing unit normal for $K \in \Omega_{h}$, and $\hat{\mathbf{n}}_{\Gamma_{h}}$ for a unit normal defined on the skeleton of the mesh. Thus for each internal face an arbitrary (between the two possibilities), but fixed throughout the computation, direction for $\hat{\mathbf{n}}_{\Gamma_{h}}$ is chosen; on $\Gamma$ it is assumed that the outwards facing unit normal for $\Omega$ is used. In this notation the ultra-weak Petrov-Galerkin formulation of (1) is stated as follows: find $U \in \mathcal{U}_{\hat{\mathbf{t}}, \hat{\mathbf{u}}_{0}}$, such that

$$
b(U, \tilde{V})=\ell(\tilde{V}), \quad \forall \tilde{V} \in \mathcal{V} .
$$

Equivalently, this problem can be stated as a mixed Bubnov-Galerkin problem: find $(U, V) \in \mathcal{U}_{\hat{\mathbf{t}}, \hat{\mathbf{u}}_{0}} \times \mathcal{V}$, such that

$$
\begin{aligned}
a(V, \tilde{V})+b(U, \tilde{V}) & =\ell(\tilde{V}), & & \forall \tilde{V} \in \mathcal{V}, \\
b(\tilde{U}, V) & =0, & & \forall \tilde{U} \in \mathcal{U}_{\mathbf{0}, \mathbf{0}},
\end{aligned}
$$

where $a: \mathcal{V} \times \mathcal{V} \rightarrow \mathbb{R}$ is an inner product on $\mathcal{V}$. Note that the problem (5) is nothing else but the optimality condition for the residual minimization problem associated with the ultra-weak statement (4), namely

$$
\operatorname{minimize}_{U \in \mathcal{U}_{\hat{\mathbf{t}}, \hat{u}_{0}}} \frac{1}{2}\|B(U, \cdot)-\ell(\cdot)\|_{\mathcal{V}^{\prime}}^{2} .
$$


Furthermore, for an arbitrary function $U \in \mathcal{U}$, Riesz representation theorem [5] guarantees us the existence of a unique $V_{U} \in \mathcal{V}$ solving the the first equation in (5). This $V_{U} \in \mathcal{V}$ is precisely the Riesz representation of the residual $B(U, \cdot)-\ell(\cdot) \in \mathcal{V}^{\prime}$ with respect to the inner product $a(\cdot, \cdot)$. Having found this representation we can easily compute the norm of the residual as $\|B(U, \cdot)-\ell(\cdot)\|_{\mathcal{V}^{\prime}}^{2}=$ $a\left(V_{U}, V_{U}\right)$.

What makes this least squares/mixed approach practical is the fact that the test space $\mathcal{V}$ is a broken Sobolev space. Therefore, the inner product $a(\cdot, \cdot)$ decomposes into a sum of element-wise integrals, and ultimately after discretization results in a block-diagonal matrix. Consequently, $V$-unknowns can be easily eliminated from (5) in an embarrassingly parallel, element-wise fashion, and the resulting Schur complement is symmetric and positive definite, regardless of the properties of the ultra-weak formulation (4) associated with the form $b(\cdot, \cdot)$ beyond its well-posedness.

Note that within this framework we have a freedom of selecting an inner product $a$ on the test space $\mathcal{V}$ as we see fit for a particular purpose. For example, in our case we could equip $\mathcal{V}$ with the standard inner product defined in the broken Sobolev space $H\left(\Omega_{h}, \operatorname{div} ; \mathbb{S}^{n}\right) \times H^{1}\left(\Omega_{h} ; \mathbb{R}^{n}\right)$ :

$$
\begin{aligned}
a_{\text {natural }}\left(V_{1}, V_{2}\right) & =\sum_{K \in \Omega_{h}}\left[\left(\nabla \mathbf{v}_{1}, \nabla \mathbf{v}_{2}\right)_{K}\right. \\
& \left.+\left(\operatorname{div} \boldsymbol{\tau}_{1}, \operatorname{div} \boldsymbol{\tau}_{2}\right)_{K}+\left(\boldsymbol{\tau}_{1}, \boldsymbol{\tau}_{2}\right)_{K}+\left(\mathbf{v}_{1}, \mathbf{v}_{2}\right)_{K}\right]
\end{aligned}
$$

resulting in what is often called "the mathematician's norm" in the DPG context. Another standard, and often better choice is to consider the graph product defined by $b$, plus the $L^{2}$ products of the test functions to make the bilinear form positive definite:

$$
\begin{aligned}
a_{\text {graph }, \varrho}\left(V_{1}, V_{2}\right) & =\sum_{K \in \Omega_{h}}\left[\left(\mathbf{S}_{\varrho} \boldsymbol{\tau}_{1}+\varepsilon\left(\mathbf{v}_{1}\right), \mathbf{S}_{\varrho} \boldsymbol{\tau}_{2}+\varepsilon\left(\mathbf{v}_{2}\right)\right)_{K}\right. \\
& \left.+\left(\operatorname{div} \boldsymbol{\tau}_{1}, \operatorname{div} \boldsymbol{\tau}_{2}\right)_{K}+\left(\boldsymbol{\tau}_{1}, \boldsymbol{\tau}_{2}\right)_{K}+\left(\mathbf{v}_{1}, \mathbf{v}_{2}\right)_{K}\right]
\end{aligned}
$$

We will also mention the "energy" inner product, defined in [6]:

$$
\begin{aligned}
a_{\text {energy }}\left(V_{1}, V_{2}\right) & =\sum_{K \in \Omega_{h}}\left[\left(\mathbf{S}_{\varrho}^{-1} \varepsilon\left(\mathbf{v}_{1}\right), \boldsymbol{\varepsilon}\left(\mathbf{v}_{2}\right)\right)_{K}\right. \\
& \left.+\left(\operatorname{div} \boldsymbol{\tau}_{1}, \operatorname{div} \boldsymbol{\tau}_{2}\right)_{K}+\left(\mathbf{S}_{\varrho} \boldsymbol{\tau}_{1}, \boldsymbol{\tau}_{2}\right)_{K}+\left(\mathbf{v}_{1}, \mathbf{v}_{2}\right)_{K}\right]
\end{aligned}
$$

Note that despite the introduction of the mesh, mesh-dependent broken spaces and norms, the problem has not yet been discretized. The ideal DPG method is defined by discretizing the least squares statement (6) by introducing a suitable finite dimensional piecewise-polynomial subspace $\mathcal{U}_{h} \subset \mathcal{U}$. Note that all elements of the solution, except for the trace $\hat{\mathbf{u}}$, may contain discontinuities which allows for a great freedom of choice of conforming elements, in particular we can use Lagrange elements to represent piecewise-continuous symmetric stress tensors. However, this is only a semi-discretization, as it corresponds to keeping the space 
$\mathcal{V}$ infinite-dimensional in (5). Practical DPG methods are obtained by selecting a sufficiently fine, when compared with $\mathcal{U}_{h}$, discretization $\mathcal{V}_{h} \subset \mathcal{V}$. A typical strategy for selecting such a $\mathcal{V}_{h}$ is to raise the power of polynomials used to approximate $(\boldsymbol{\sigma}, \mathbf{u})$ locally on each element $K \in \Omega_{h}$.

We have implemented this strategy using a finite element library deal.II [3]. In this implementation $\Omega$ is subdivided into quadrilateral or hexagonal elements, which are mapped to reference elements using the standard bi/trilinear maps. On each reference square/cube, each component of $(\boldsymbol{\sigma}, \mathbf{u})$ is discretized using tensor product of polynomials of degree less than or equal to $p \geq 0$ in each coordinate direction, without assuming any interelemental continuity. Each component of $\hat{\boldsymbol{\sigma}}$ is similarly discretized by tensor product of polynomials of degree less than or equal to $p \geq 0$ on each reference interval/square. Each component of $\hat{\mathbf{u}}$ is discretized with tensor product polynomials of degree $p+1$ on a reference square/interval, and we have to assume global continuity on $\Gamma_{h}$ of these approximations to conform to the space $H^{1 / 2}\left(\Gamma_{h} ; \mathbb{R}^{n}\right)$. Finally, $(\boldsymbol{\tau}, \mathbf{v})$ are discretized in the same fashion as $(\boldsymbol{\sigma}, \mathbf{u})$, but with polynomial degree raised to $p+\Delta p$, where we use $\Delta p=2$ in our implementation. As mentioned previously we eliminate the degrees of freedom associated with $(\boldsymbol{\tau}, \mathbf{v})$ when performing an elemental assembly in order to obtain a smaller symmetric positive definite system instead of the symmetric indefinite system resulting directly from (5). Additionally, we also eliminate the degrees of freedom associated with $(\boldsymbol{\sigma}, \mathbf{u})$ on each element (static condensation) to further reduce the size of the global system. Thus the final algebraic system only contains the global unknowns $(\hat{\boldsymbol{\sigma}}, \hat{\mathbf{u}})$ defined on the skeleton of the mesh.

In fact, we also utilize the lowest-order DPG $(p=0)$ to discretize the Helmholtz problem associated with the filtering operator in our implementation, so that both $\rho$ and $\varrho=\mathcal{F}_{r} \rho$ are approximated with piece-wise constants on each element. However, we only explain the details for the elasticity system in this document.

\section{Convergence test}

We verify the correctness of our implementation of the DPG method using the method of manufactured solutions, see e.g. [13]. Again, here we focus on the verification of the elasticity "part" of the code. The linear algebraic system resulting from the DPG discretization is solved using using a direct solver ( $\mathrm{Su}-$ perLU_dist [11], available in deal.II via Trilinos [9] wrappers) in order to focus on the discretization error only. We consider a smooth $2 \mathrm{D}$ problem corresponding to $E=2.0, \nu=0.3, \mathbf{u}_{\text {an. }}=(\sin (\pi(x+y)), \sin (\pi y))$ on a unit square with a mixture of displacement and traction boundary conditions. The problem is solved on a sequence of globally refined grids, starting with an unstructured coarse grid containing only 12 quadrilateral cells obtained using GMSH [8]. Various measures of error, including the norm of the residual in $\mathcal{V}^{\prime}$, are summarized in Figure 1 for the case of the graph inner product on the test space $\mathcal{V}$, see (8). Note that in all cases the optimal convergence rate of $O\left(h^{p+1}\right)$ in both stress and displacement 
components is observed. Other inner products, such as (7) and (9) demonstrate nearly identical behaviour in this case.

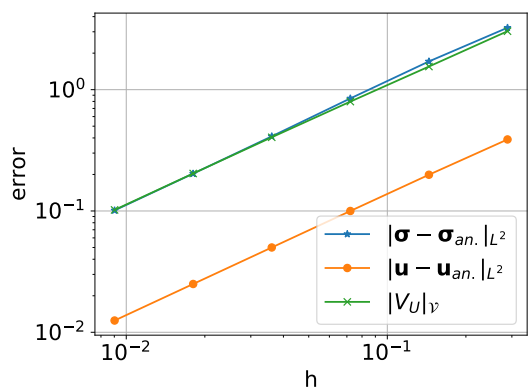

(a)

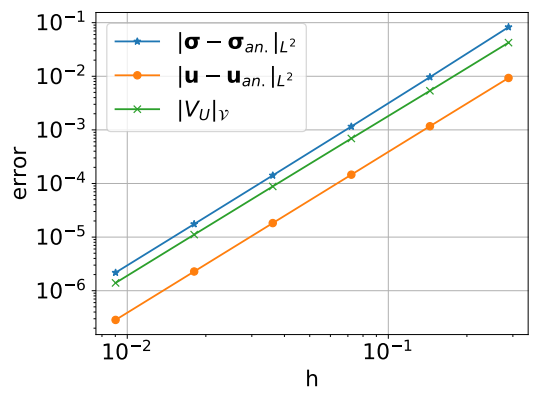

(c)

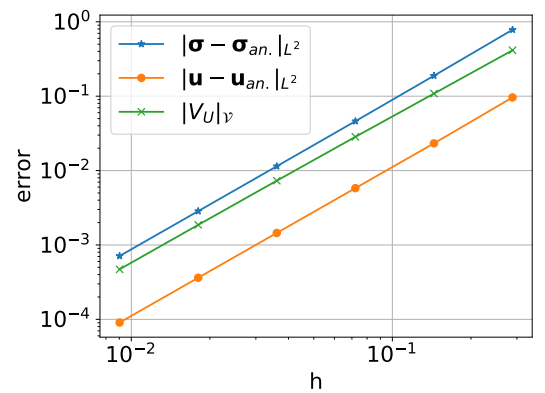

(b)

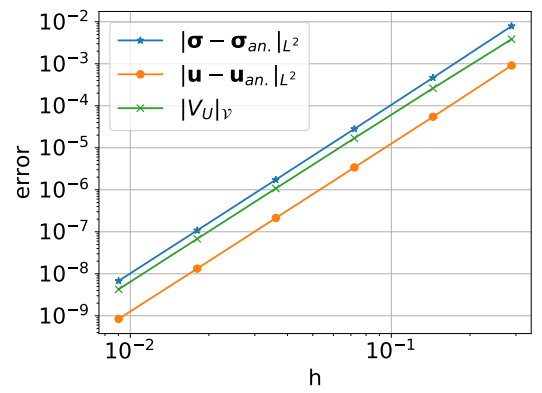

(d)

Fig. 1. Convergence test of the implemented DPG method. (a): $p=0 ;(\mathrm{b}): p=1 ;(\mathrm{c})$ : $p=2 ;(\mathrm{d}): p=3$.

\section{Performance of the DPG method on problems with jumps in the coefficients}

Having verified the implementation of the finite element discretization, we test the method on a standard "cantilever" topology optimization benchmark [4], see Figure 2. Note that the "standard" test space inner products outlined above result in rather strange material distributions, which do not reproduce the wellestablished benchmark.

To pinpoint the cause of this issue, we again utilize the method of manufactured solutions, but now on a boundary value problem with a jump in the coefficients. To further simplify the problem we consider a scalar problem, which is obtained from the elasticity equations we have presented by setting $\nu=0$ and putting all the problem data (volumetric and traction loads, boundary displacements) corresponding to one spatial dimension to 0 . We consider a problem 


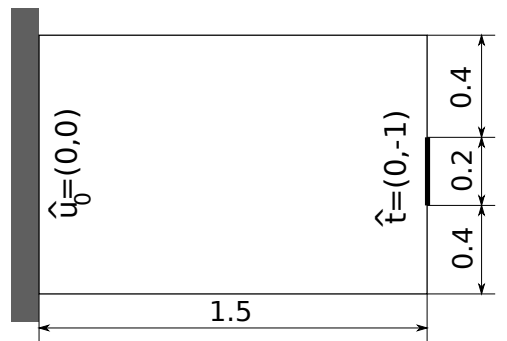

(a)

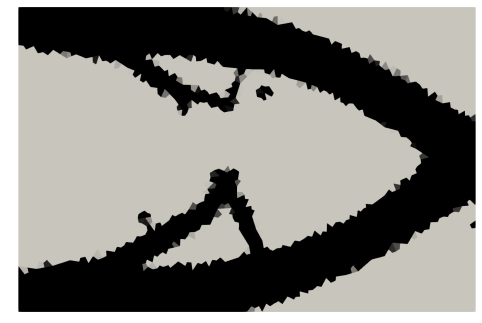

(c)

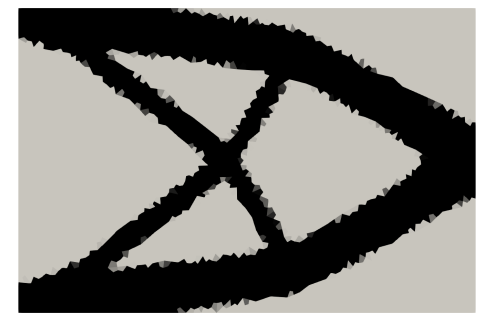

(e)

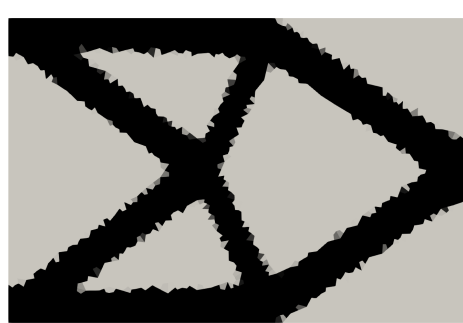

(b)

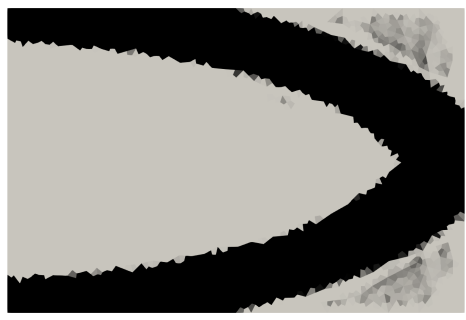

(d)

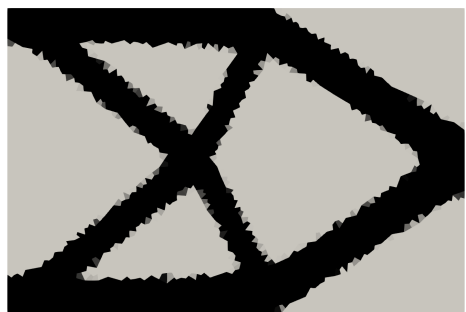

(f)

Fig. 2. Performance of the DPG method on a "cantilever" topology optimization benchmark. (a): definition of the supports and loads; (b): reference solution obtained using standard conforming Galerkin FEM (based on the displacement only formulation, using bilinear elements); (c): DPG FEM solution with the inner product (7); (d): DPG FEM solution with the inner product (8); (e): DPG FEM solution with the inner product (9); (f): DPG FEM solution with the properly scaled inner product (10). 
defined on a unit square $\Omega=(-1,1)^{2}$, which is subdivided into two subdomains defined in polar coordinates as follows: $\Omega_{1}=\{(r \cos (\theta), r \sin (\theta)) \in \Omega \mid r>$ $0,|\theta|<\hat{\theta}\}$, and similarly $\Omega_{2}=\{(r \cos (\theta), r \sin (\theta)) \in \Omega|r>0, \hat{\theta}<| \theta \mid \leq \pi\}$ for some $0<\hat{\theta}<\pi$. We set $E=E_{1}$ on $\Omega_{1}$ and $E=E_{2}$ on $\Omega_{2}$. The solution (displacement) is constructed in polar coordinates as $u_{1 \text {,an. }}(r, \theta)=r^{k} p(\theta), u_{2, \text { an. }}(r, \theta) \equiv 0$, where $p(\theta)$ is a cubic polynomial on each subdomain. This spline is constructed in such a way that $p(0)=-p( \pm \pi)=\hat{C}, p( \pm \hat{\theta})=0, p^{\prime}(0)=p^{\prime}( \pm \pi)=0$, and finally the jump in its normal derivatives across the subdomain boundaries is determined by the ratio $E_{1} / E_{2}$, as the normal stresses must be continuous across the subdomain boundary. Namely, on the subdomain 1 we put

$$
p(\theta)=\hat{C}+|\theta|^{2}\left[\hat{\theta}^{-3}\left(2 \hat{C}-\hat{\theta} E_{2}\right)|\theta|+\hat{\theta}^{-2}\left(\hat{\theta} E_{2}-3 \hat{C}\right)\right]
$$

and symmetrically on subdomain 2. GMSH is used to produce a grid conforming to the subdomain subdivision, and the problem is solved on a sequence of globally refined grids. In our test we put $\hat{\theta}=\pi / 3, k=2, \hat{C}=1, E_{1}=10^{0}$, $E_{2}=10^{-9}$. That is, the contrast ratio in this test corresponds to the standard settings in topology optimization applied to linear elasticity problems. We measure and report the behaviour of stresses and displacements on each subdomain in Figure 3 (a). Only a representative case of the inner product (8) is shown, other

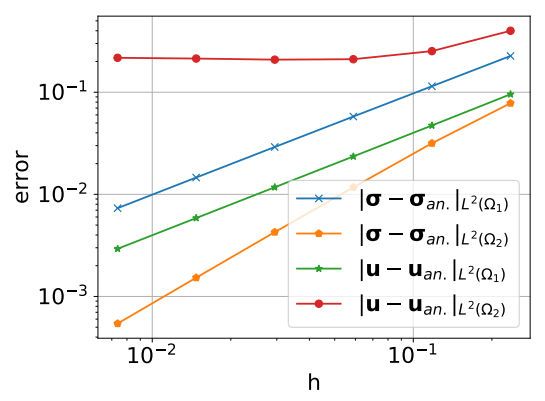

(a)

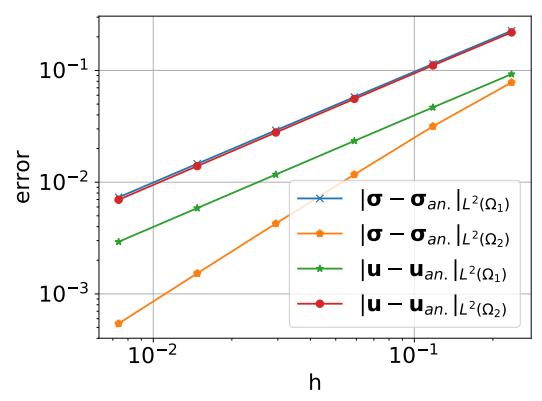

(b)

Fig. 3. Performance of the DPG method on a representative problem with jumps in the coefficients. (a): DPG FEM solution with the inner product (8); (b): DPG FEM solution with the properly scaled inner product (10).

inner products mentioned above result in nearly identical behaviour. Note that whereas the stresses on subdomain 2 demonstrate apparent superconvergence with the rate $O\left(h^{1.5}\right)$, it should be understood in the context of the fact that stresses in subdomain 2 are close to 0 because of the very small Young's modulus there. Additionally, the displacements in this subdomain do not converge at all.

The inner product (9) hints how the norm on the test space $\mathcal{V}$ should be scaled: note that it performs the best out of the DPG methods in Figure 2 and in fact it behaves much better on finer grids. This indicates that if in addition to 
scaling the derivative parts of the test inner product we also scale the $L^{2}$ parts, we may recover the convergence of the method on problems with big jumps in the coefficients. We illustrate this idea by scaling the graph inner product (8); other products, when scaled, demonstrate similar performance. Thus we put

$$
\begin{aligned}
a_{\text {scaled } \varrho}\left(V_{1}, V_{2}\right) & =\sum_{K \in \Omega_{h}}\left[E_{\varrho}\left(\mathbf{S}_{\varrho} \boldsymbol{\tau}_{1}+\varepsilon\left(\mathbf{v}_{1}\right), \mathbf{S}_{\varrho} \boldsymbol{\tau}_{2}+\boldsymbol{\varepsilon}\left(\mathbf{v}_{2}\right)\right)_{K}\right. \\
& \left.+E_{\varrho}^{-1}\left(\operatorname{div} \boldsymbol{\tau}_{1}, \operatorname{div} \boldsymbol{\tau}_{2}\right)_{K}+E_{\varrho}^{-1}\left(\boldsymbol{\tau}_{1}, \boldsymbol{\tau}_{2}\right)_{K}+E_{\varrho}\left(\mathbf{v}_{1}, \mathbf{v}_{2}\right)_{K}\right],
\end{aligned}
$$

where the piecewise-constant Young's modulus $E_{\varrho}$ in our case obeys the SIMP law $E_{\varrho}=E_{1} \varrho^{p}$, with $E_{1}$ being the Young's modulus of the stiff material. From Figure 3 (b) one can see that indeed the optimal convergence rates are recovered when the scaled inner product is utilized. Furthermore, the topology optimization algorithm based on the DPG discretization of the elasticity problem behaves in almost exactly (up to the discretization error, of course) the same fashion as its conforming continuous Galerkin counterpart, see Figure 1 (b) and (f), where only the final design is shown.

\section{Premature termination of Krylov iteration based on the residual estimate}

As we mentioned, DPG discretizations result in symmetric and positive definite matrices, for which the conjugate gradient iteration is a preferred solution algorithm. In the present case, the $\mathrm{CG}$ iteration is in fact equivalent to the residual minimization, as can be easily seen from the following calculation. Indeed, let us define the bounded linear operators $B: \mathcal{U} \rightarrow \mathcal{V}^{\prime}$ and $A: \mathcal{V} \rightarrow \mathcal{V}^{\prime}$ by $B U(V)=b(U, V)$ and $A V_{1}\left(V_{2}\right)=a\left(V_{1}, V_{2}\right)$. Let further $S=B^{\prime} A^{-1} B \in \mathcal{L}\left(\mathcal{U}, \mathcal{U}^{\prime}\right)$ and $d=B^{\prime} A^{-1} \ell \in \mathcal{U}^{\prime}$ be the left hand side and the right hand side of the problem (5) obtained after the unknown $V$ is eliminated. Then the function $(0.5 S U-d)(U)$ minimized by the CG algorithm applied to the problem $S U=d$ equals to

$$
\begin{aligned}
(0.5 S U-d)(U) & =\left[B^{\prime} A^{-1}(0.5 B U-\ell)\right](U)=b\left(U, A^{-1}(0.5 B U-\ell)\right) \\
& =[B U]\left(A^{-1}(0.5 B U-\ell)\right)=(B U, 0.5 B U-\ell)_{\mathcal{V}^{\prime}} \\
& =0.5\|B U-\ell\|_{\mathcal{V}^{\prime}}^{2}-0.5\|\ell\|_{\mathcal{V}^{\prime}}^{2}
\end{aligned}
$$

where the equality in line two of the chain of equalities above is owing to the Riesz' representation theorem. Note that since the term $\|\ell\|_{\mathcal{V}^{\prime}}^{2}$ is independent from $U$, minimizing $(0.5 S U-d)(U)$ is equivalent with minimizing $\|B U-\ell\|_{\mathcal{V}^{\prime}}^{2}$. The same conclusion carries through to the discretization process, we only need to replace the spaces $\mathcal{U}$ and $\mathcal{V}$ with the conforming finite dimensional subspaces. The only difference is that instead of minimizing the true residual $\|B U-\ell\|_{\mathcal{V}^{\prime}}^{2}$ of the ultra-weak formulation of the elasticity PDE we would minimize its estimate. Note that even at the exact solution of the discretized equations this residual estimate is not going to be zero, but rather contain terms related to 
the discretization error. Thus it is natural to stop the CG iteration when the residual estimate $\eta=\|B U-\ell\|_{\mathcal{V}^{\prime}}$ is not changing any longer, at which point we conclude that discretization error terms dominate the residual and further progress will not bring us closer to the true solution of the governing PDE. Such a strategy has been utilized in [12]. Namely, let $\eta_{k}$ be the residual estimate at the $k$ th Krylov iteration. Then we stop the iteration as soon as the relative change $\left|\eta_{k}-\eta_{k-1}\right| / \eta_{k}<\varepsilon$, where $\varepsilon$ is some prescribed tolerance. In our numerical experiments we put $\varepsilon=10^{-4}$. For comparison, we also include the results obtained with a direct solver, and with an the standard PCG stopping criterion based on the Euclidean norm of the discretized residual being less than $10^{-6}$. Black box algebraic mutligrid preconditioner MueLu from Trilinos is utilized via deal.II wrappers (and arguably it does not work very well in this fashion for our problem). The results are reported in Figure 4, where all graphs corresponding to the direct solver are marked with "LU", those with the premature termination of PCG based on the residual estimate with "V", and the standard stopping of PCG iteration with "CG".

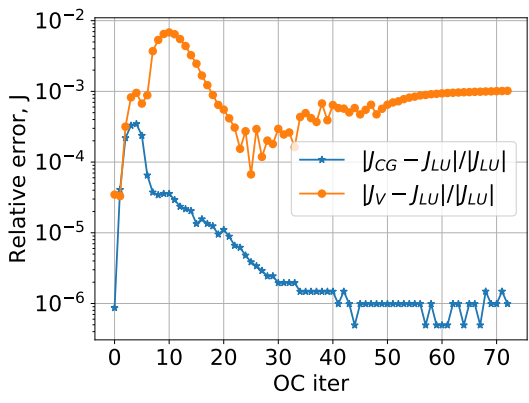

(a)

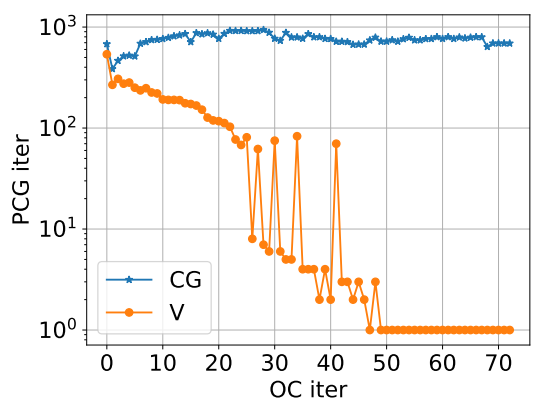

(c)

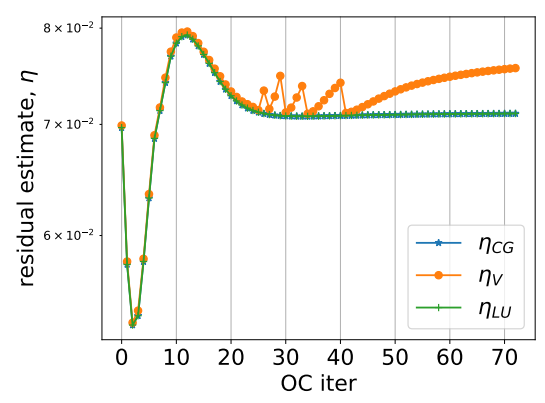

(b)

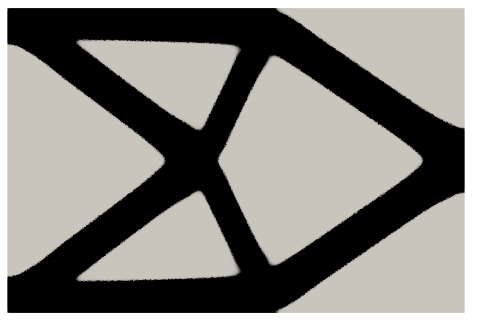

(d)

Fig. 4. Performance of the DPG method with early termination of PCG iteration on a representative problem with jumps in the coefficients. (a): relative difference in the objective function values; (b): relative residual estimate of the elasticity equations; (c): number of PCG iterations; (d): final design obtained using premature PCG iteration. 


\section{Conclusion and discussion}

Our preliminary findings confirm the possibility of utilizing properly scaled DPG FEM within topology optimization, which provides us with a "free" a posteriori residual estimates. These can be utilized for example for premature termination of Krylov solvers as illustrated here, or within the context of adaptivity, which is one of our current research directions.

\section{References}

1. Allaire, G.: Shape optimization by the homogenization method, vol. 146. Springer Science \& Business Media (2012)

2. Amir, O., Sigmund, O.: On reducing computational effort in topology optimization: How far can we go? Struct. Multidiscip. Optim. 44(1), 25-29 (2011). DOI 10.1007/ s00158-010-0586-7

3. Bangerth, W., Hartmann, R., Kanschat, G.: deal.II - a general purpose object oriented finite element library. ACM Trans. Math. Softw. 33(4), 24/1-24/27 (2007)

4. Bendsøe, M.P., Sigmund, O.: Topology optimization: theory, methods, and applications, 2 edn. Springer-Verlag Berlin Heidelberg (2004). DOI 10.1007/ 978-3-662-05086-6

5. Brezis, H.: Functional analysis, Sobolev spaces and partial differential equations. Springer Science \& Business Media (2010)

6. Carstensen, C., Hellwig, F.: Low-order discontinuous Petrov-Galerkin finite element methods for linear elasticity. SIAM J. Numer. Anal. 54(6), 3388-3410 (2016). DOI $10.1137 / 15 \mathrm{M} 1032582$

7. Demkowicz, L., Gopalakrishnan, J.: A class of discontinuous Petrov-Galerkin methods. II. optimal test functions. Numerical Methods for Partial Differential Equations 27(1), 70-105 (2011)

8. Geuzaine, C., Remacle, J.F.: Gmsh: A 3-d finite element mesh generator with builtin pre-and post-processing facilities. International journal for numerical methods in engineering 79(11), 1309-1331 (2009)

9. Heroux, M., Bartlett, R., Hoekstra, V.H.R., Hu, J., Kolda, T., Lehoucq, R., Long, K., Pawlowski, R., Phipps, E., Salinger, A., Thornquist, H., Tuminaro, R., Willenbring, J., Williams, A.: An Overview of Trilinos. Tech. Rep. SAND2003-2927, Sandia National Laboratories (2003)

10. Keith, B., Fuentes, F., Demkowicz, L.: The dpg methodology applied to different variational formulations of linear elasticity. Computer Methods in Applied Mechanics and Engineering 309, 579-609 (2016)

11. Li, X.S., Demmel, J.W.: Superlu_dist: A scalable distributed-memory sparse direct solver for unsymmetric linear systems. ACM Transactions on Mathematical Software (TOMS) 29(2), 110-140 (2003)

12. Limkilde, A., Evgrafov, A., Gravesen, J.: On reducing computational effort in topology optimization: We can go at least this far! (2018). Revised for Struct. Multidiscip. O.

13. Roache, P.J.: Code verification by the method of manufactured solutions. Journal of Fluids Engineering 124(1), 4-10 (2002) 